\title{
Submicron Spatial Resolution in Thermal Desorption Mass Spectrometry via Rapid Heating Functions using Thermal AFM Probes.
}

\author{
Suhas Somnath $^{1}$, Stephen Jesse ${ }^{1}$, Gary J. Van Berkel ${ }^{2}$, Sergei V. Kalinin ${ }^{1}$, and Olga S. Ovchinnikova ${ }^{2}$ \\ 1. The Institute for Functional Imaging of Materials and the Center for Nanophase Materials Sciences, \\ Oak Ridge National Laboratory, Oak Ridge, TN 37831, USA. \\ 2. Mass Spectrometry and Laser Spectroscopy Group, Chemical Sciences Division, Oak Ridge National \\ Laboratory, Oak Ridge, TN 37831, USA.
}

Atomic Force Microscopy (AFM) combined with Mass Spectrometry (MS) can provide the ability to map and correlate the molecular and physical properties of samples $[1,2]$ at sub-micron resolutions. Our group has demonstrated such a platform using heated AFM probes [3] for the thermal desorption (TD) of molecules. Current heating techniques typically result in a minimum desorption crater diameter of 1-2 $\mu \mathrm{m}$ [2]. Crater sizes are even larger for materials where the melting and vaporization points are further apart since the majority of the thermal energy from the probe only melts or damages the substrate. Prior research shows that rapid heating (faster than $10^{9} \mathrm{~K} / \mathrm{s}$ ) is necessary for TD of large intact molecules [4]. Here, we will discuss the investigation and implementation of successful strategies to maximize a figure-of-merit, defined as the quotient of the MS signal level and the desorption crater diameter, by tailoring the heating pulse waveform in the AFM probe to maximize the TD efficiency. These improvements result in heating rates approaching $10^{9} \mathrm{~K} / \mathrm{s}$ which translates directly to enhanced spatial resolution and sensitivity of chemical species.

A Thermo Instruments LTQ-XL MS with a vapor transfer line, in a "T" geometry, with an Atmospheric Pressure Chemical Ionization (APCI) corona needle in one arm of the "T", was used as in our previous reports [1]. Test samples consisting of pigment yellow 74 were printed onto paper by an inkjet printer. We used an Anasys Instruments ThermaLever AN300 cantilever probe, which has an integrated resistive heater over the probe tip. The probe was mounted in an Anasys Instruments AFM+ such that the probe tip was in contact with the sample, and positioned $\sim 0.5 \mathrm{~mm}$ from the entrance of the MS capillary. The probe was heated using custom Labview software via National Instruments data acquisition (DAQ) boards. The vacuum draw from the MS pulled the vapors from the AFM region into the mass spectrometer through the capillary.

In TD-MS AFM, voltage is applied to the thermal probe to induce joule-heating, which raises the temperature of the probe tip causing TD of molecules off the sample. We can control the heating rate of the probes from $10^{2}$ to $10^{9} \mathrm{~K} / \mathrm{s}$ and achieve temperatures of 300 to $1300 \mathrm{~K}$ by varying the magnitude of the heating voltage. In the traditional heating method, referred to as scheme A, the probe is heated by a square voltage pulse of height, $V_{2}$, for duration, $t_{2}$ as seen in figure $1(\mathrm{a}, \mathrm{d}, \mathrm{g})$. Increasing $V_{2}$ and decreasing $t_{2}$ shrunk the desorption craters from $3.5 \mu \mathrm{m}$ to $1.8 \mu \mathrm{m}$ in diameter and raised the figure-ofmerit from 0.4 to 2.7. Figure $1(\mathrm{~b}, \mathrm{e}, \mathrm{h})$ shows heating scheme $\mathrm{B}$, where we added another pulse with considerably larger magnitude $\left(V_{l}\right)$ but shorter duration $\left(t_{1}\right)$ before the main heating pulse $\left(V_{2}, t_{2}\right)$. This scheme improved the heating rate and the duration at which the probe was at the target temperature. Though the figure-of-merit of scheme $\mathrm{B}$ improved by $43 \%$ compared to scheme $\mathrm{A}$ for short $t_{2}$, it was challenging to control the heating for longer $t_{2}$. Figure $1(\mathrm{~d}, \mathrm{e})$ show that both schemes result in significant re-deposition of desorbed molecules and reflow of melted material around the craters. Thus, the effective spatial resolution for these schemes is larger than the crater dimensions. In order to 
maximize TD, and minimize the damaged surface area, the probe needs to be heated rapidly past the melting and sublimation point. This was accomplished in heating scheme $\mathrm{C}$, where $V_{1}$ and $V_{2}$ are increased significantly while $t_{1}$ and $t_{2}$ are decreased, and these pulses are repeated 5-10 times as shown in figure 1(c, f, i). Through this scheme, the desorption craters shrunk to $400-600 \mathrm{~nm}$ in diameter and the figure-of-merit rose to 10.2. Furthermore, figure 1(f) shows that the re-deposition and reflow of the substrate were negligible and the majority of the material was desorbed. These $400 \mathrm{~nm}$ wide craters are close to the sensitivity limit of our mass spectrometer, and further improvements in spatial resolution would require improvements in mass transport, ionization or mass spectrometer detection. We are studying other materials including poly(2-vinylpyridine) and rubrene to investigate the material types that will allow nanometer scale sampling with the new heating techniques.

This research was funded by the United States Department of Energy, Office of Science, Basic Energy Sciences, Chemical Sciences, Geosciences, and Biosciences Division [GJV,OSO] and the Center for Nanophase Materials Sciences, which is a U. S. Department of Energy Office of Science User Facility $[\mathrm{SS}, \mathrm{SJ}, \mathrm{SVK}]$. We thank Anasys Instruments for providing thermal probes and the AFM for this research.

References:

[1] O. S. Ovchinnikova, et al., ACS Nano 9 (2015), p. 4260-4269.

[2] O. S. Ovchinnikova, et al., Analytical Chemistry 86 (2013), p. 1083-1090.

[3] W. P. King, et al., Annual Review of Heat Transfer 16 (2013), p. 287-326.

[4] D. T. Usmanov, et al., Journal of The American Society for Mass Spectrometry 24 (2013), p. 17271735.
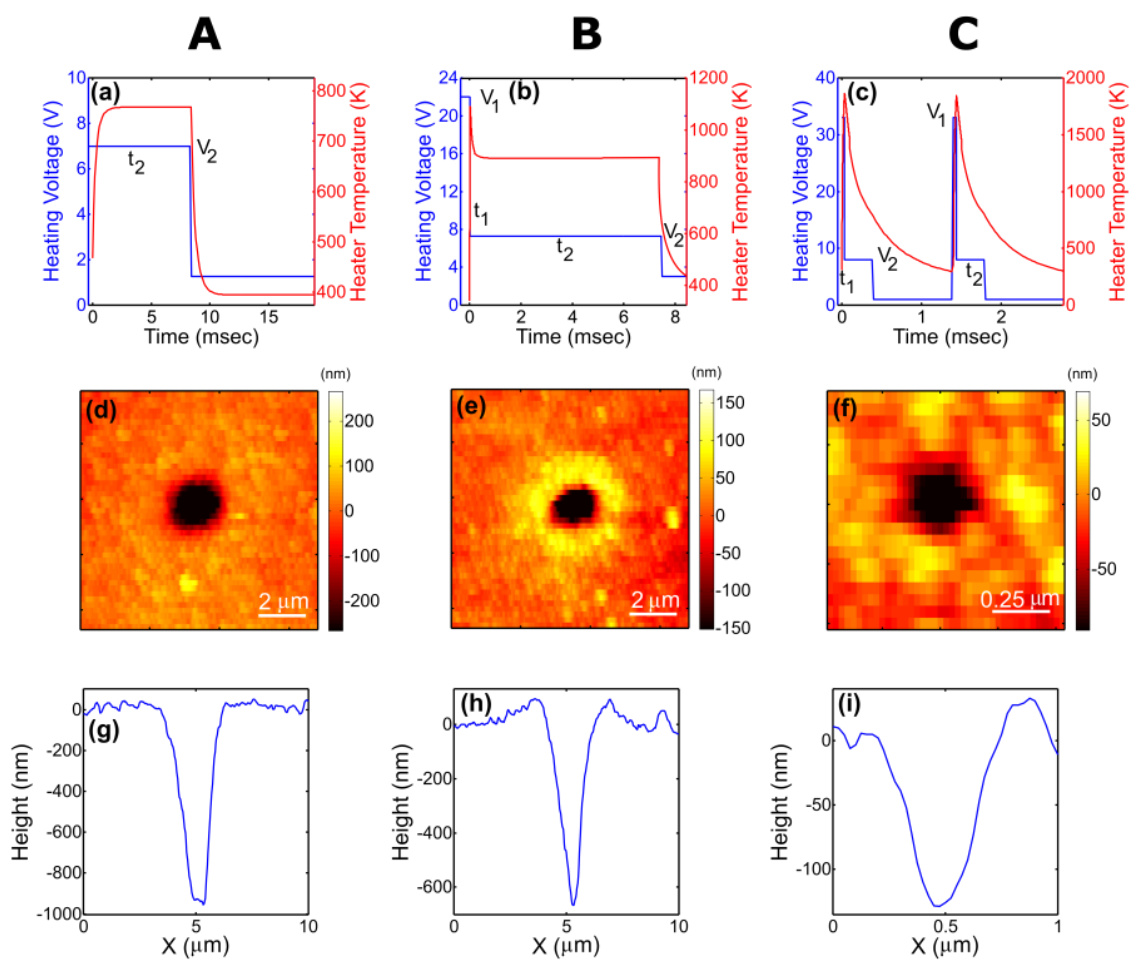

Figure 1. (a-c) Heating voltages and probe heater temperatures as a function of time for different heating schemes. (d-f) Topography maps of, and (g-i) line scans at the center of the desorption craters. 